\title{
Air-travel study struggles to get airborne
}

Declan Butler, Paris

A partial rescue plan has been agreed for the first large-scale study of whether passengers on long-haul flights are at increased risk of developing life-threatening blood clots.

Scientists have obtained only around a quarter of the 12 million euros (US\$11 million) needed for the project, but they nonetheless aim to launch it this week, while continuing to search for the remaining funds.

The past month has seen a spate of lawsuits against airlines by victims of deep-vein thrombosis (DVT) - 'economy-class syndrome' - and their relatives. Proper scientific data are needed to improve our understanding of the risks involved so that we can offer sound safety advice for air travel, says William Toff, a cardiologist at the University of Leicester and a member of the project team.

The proposed project is far larger than previous studies of the problem. Its scientific protocol - called the WHO Research Initiative on Global Hazards of Travel (WRIGHT) - was approved by the World Health Organisation (WHO) last August.

The International Air Transport Associa-

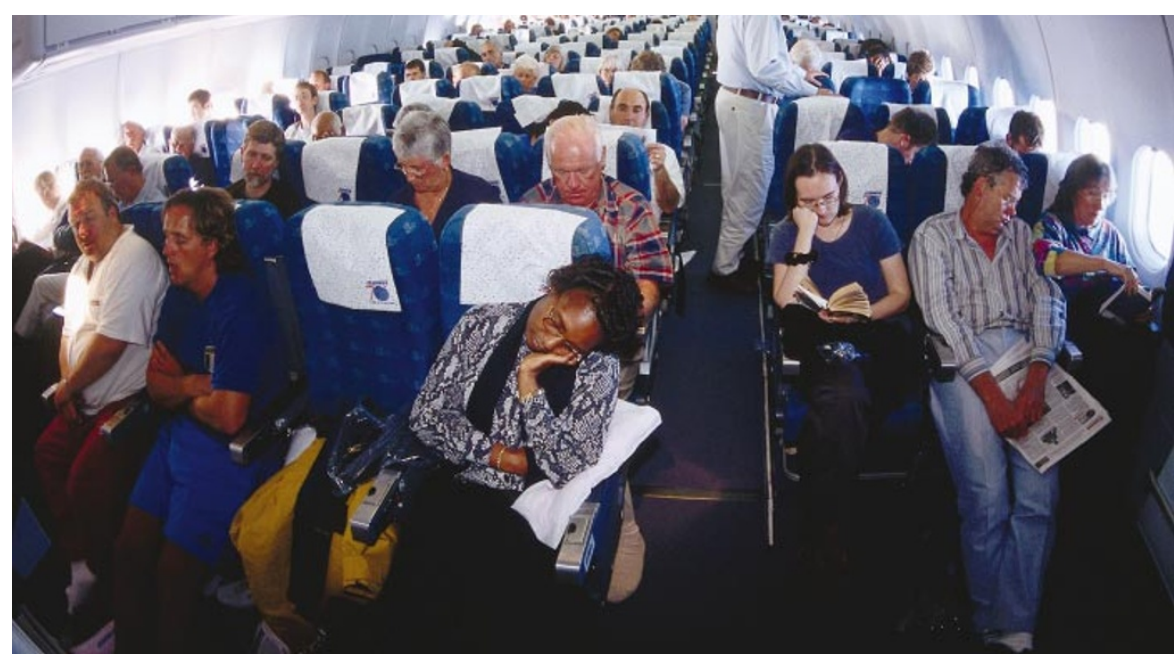

Up in the air: a proposed study would involve passengers on 200,000 flights — if funding can be found.

tion, which represents the airlines, has welcomed the study — at least in public — and has agreed to help the WHO and the International Civil Aviation Organization with the logistics involved.

Fund-raising is being done by the WHO,

\section{Canadian lab loses Amgen backing}

Jonathan Knight, San Francisco

A Californian biotechnology company is pulling the plug on a successful mousegenetics institute at the University of Toronto.

The withdrawal of support from the Amgen Institute is the latest indication that biotechnology and pharmaceutical firms faced with declining profits and empty drug pipelines - are losing some of their appetite for backing basic university research.

The six laboratories at the institute were considered to be exceptionally productive (see Nature 411, 519-520; 2001). But an agreement this week between Amgen, based in Thousand Oaks, California, and Toronto's University Health Network hands the administration of the institute to the Ontario Cancer Institute (OCI) in Toronto.

Although the financial details of the agreement have not been made public, Tak Mak, the Amgen Institute's director, says it will mean a sharp decrease in support from the Californian company. The institute's researchers will resort to individual grants for most of their funding, he says.

Since the institute's birth in 1993, its 50 or so scientists and technicians have been employed by Amgen, giving the company control of any intellectual property rights arising from their discoveries.

The move is part of a larger restructuring at Amgen, one of the oldest and largest biotechnology firms, with 7,000 employees and sales last year of $\$ 4$ billion. The reshuffle is expected to align research more directly with product development.

Amgen will continue to provide support "at a level appropriate to the new arrangement”, says Roger Perlmutter, Amgen's vicepresident of research and development. It is estimated that Amgen's previous support amounted to around $\$ 8$ million per year.

Christopher Paige, vice-president of research at the OCI, says there are no plans to reduce the size of the research group. "We think the Amgen Institute is one of the best groups in the community," he says.

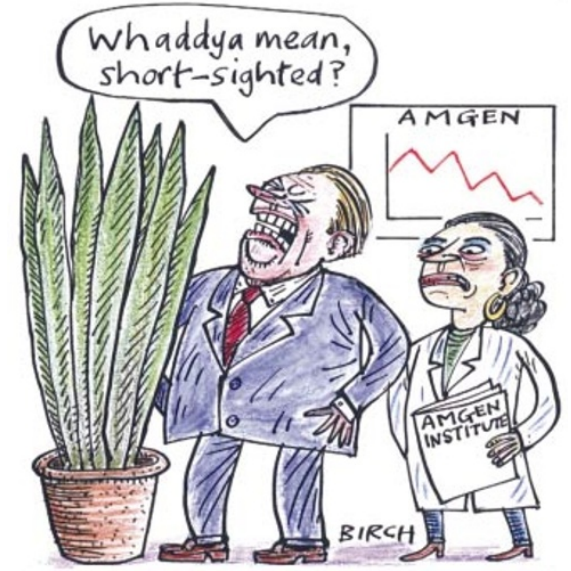

but only 2.8 million euros has been raised so far, all from within the European Union. Frits Rosendaal, a researcher at Leiden University in the Netherlands, resigned from the project last month, citing a "lack of confidence in the complete commitment from the WHO".

Shanthi Mendis, a Geneva-based WHO official involved in project planning, says the WHO is "disappointed" that funding has not materialized. "We expected a more enthusiastic response from governments," she says. But several DVT researchers blame bureaucracy at the WHO for the lack of uptake.

At a meeting in Geneva last week of the project's scientific committee and its European funders, DVT researchers said that they would try to bypass the $\mathrm{WHO}$ and solicit funding directly from governments and other sources. To safeguard the study's independence, airlines will not be asked for money.

The meeting agreed that the first two phases of the study will begin this month. One will examine the role of passenger risk factors, such as alcohol consumption, seating conditions and predisposition to the disease. The second will involve the use of experimental pressurized cabins to determine whether low air pressure during flights contributes to the risks of sitting in cramped conditions for long periods.

In the final, most expensive phase, the study would track the health of passengers on some 200,000 flights. But this phase will begin only when full funding has been obtained. And the prospects for that have worsened following the decision by Australia - the nation that arguably depends most on long-haul flights - to snub the WHO effort and pursue its own study.

"We are all impatiently waiting for the funds, with our troops ready to go," says Bo Eklof, chief of the Straub Foundation's Vascular Center at the University of Hawaii. 